\title{
Improvements of Statistical Learning Skills Allow Older Children to Go Beyond Single-Hypothesis Testing When Learning Words
}

\author{
Ming Yean SIA ${ }^{1}$ and Julien MAYOR ${ }^{2}$ \\ ${ }^{1}$ The University of Nottingham Malaysia Campus, Jalan Broga, 43500 Semenyih, Selangor, Malaysia and \\ ${ }^{2}$ University of Oslo, Forskningsveien 3A, 0373 Oslo, Norway \\ Address for correspondence: julien.mayor@psykologi.uio.no
}

(Received 27 November 2019; revised 31 August 2020; accepted 9 June 2021;

first published online 14 September 2021)

\begin{abstract}
Children learn words in ambiguous situations, where multiple objects can potentially be referents for a new word. Yet, researchers debate whether children maintain a single wordobject hypothesis - and revise it if falsified by later information - or whether children establish a network of word-object associations whose relative strengths are modulated with experience. To address this issue, we presented 4- to 12-year-old children with sets of mutual exclusivity (fast-mapping) trials: offering them with obvious initial hypotheses (that the novel object is the referent for the novel word). We observe that children aged six years and above, despite showing a novelty bias and retaining this novel word - novel object association, also formed an association between the novel word and the NAME-KNOWN object, thereby suggesting that older children attend to more than one word-object association, in a manner similar to associative learning. We discuss our findings in the context of competing theoretical accounts related to word learning.
\end{abstract}

Keywords: early word learning; associative process; language acquisition; cross-situational statistical learning; non-selectivity

\section{Introduction}

Over their first few years of life, children acquire a large vocabulary. While word learning situations can take many forms, researchers have invested considerable effort uncovering learning mechanisms that children, and adults, employ in ambiguous situations. Such situations, for example, arise when multiple objects act as potential referents for a novel word, a situation described as possessing referential ambiguity, or offering a mapping problem (Quine, 1960). Researchers have described how children and adults can integrate multiple ambiguous situations to establish stable word-object mappings; a situation described as cross-situational learning (CSL). Yet, the mechanisms underlying cross-situational learning are the focus of an 
intense debate; do children, and adults, keep track of multiple potential word-object associations, as described in Associative LEARning accounts (AL; Dautriche \& Chemla, 2014; Smith \& Yu, 2008; Suanda, Mugwanya, \& Namy, 2014; Vlach \& Johnson, 2013; Yu \& Smith, 2007), do they keep track of only one single candidate referent at any time, following HYPOTHESIS TESTING accounts (HT; Aravind, de Villiers, Pace, Valentine, Golinkoff, Hirsh-Pasek, Iglesias \& Sweig Wilson, 2018; Berens, Horst, \& Bird, 2018; Markman, 1990; Medina, Snedeker, Trueswell, \& Gleitman, 2011; Trueswell, Medina, Hafri, \& Gleitman, 2013), or do they use elements of both, depending on memory and attention resources (Yurovsky \& Frank, 2015)?

Under the HT perspective, word learners establish a single initial mapping between a word and an object. This initial mapping is maintained (or confirmed) unless it is falsified by subsequent evidence - a PROPOSE-BUT-VERIFY account (Medina et al., 2011; Trueswell et al., 2013), recently supported with neuroimaging evidence (Berens et al., 2018). This account has been extended to two- and three-year-olds (Woodard, Gleitman, \& Trueswell, 2016) and suggests that children, like adults, store just one hypothesised referent for each word. This approach also echoes early views on Mutual Exclusivity (ME; Markman, 1990; Markman \& Wachtel, 1988), that young children "fast-map" novel names to novel objects by initially rejecting additional associations to a name-known object, and that "mutual exclusivity would result from a more general principle, either a one-to-one mapping principle (Slobin, 1973) or the uniqueness principle (Pinker, 1984; Wexler \& Culicover, 1980) applied to word learning" (Markman \& Wachtel, 1988, p.149).

This strict interpretation of ME has since been nuanced, as evidence suggests that ME should rather be seen as a tendency, modulated by communicative context or language exposure (e.g., Byers-Heinlein \& Werker, 2009; Houston-Price, Caloghiris, \& Raviglione, 2010; Kalashnikova, Escudero, \& Kidd, 2018; Kalashnikova, Mattock, \& Monaghan, 2015; Malone, Kalashnikova, \& Davis, 2016), rather than a rule or a hard constraint. Similarly, HT perspectives suggest that, while young children may form a single initial mapping between a word and a referent, this initial mapping should be rejected if new evidence indicates it should be falsified. These perspectives converge insofar as, in absence of counterevidence, a SINGLE mapping between a word and a reference is expected.

In contrast, $\mathrm{AL}$ accounts suggest that children (Smith \& Yu, 2008; Suanda et al., 2014; Vlach \& Johnson, 2013) and adults (Dautriche \& Chemla, 2014; Yu \& Smith, 2007) form and maintain multiple associations between words and referents. The strengths and relative importance of these associations evolve across trials and are updated as additional evidence (e.g., co-occurrence between words and objects) is being accumulated. From this perspective, word learners maintain a NETWORK of word-object associations, rather than single mappings between words and objects, as posited by HT accounts.

Various studies have attempted to characterise the developmental trajectories associated with ME and AL. An increase in children's usage of ME as they grow older has been documented (although see Sia \& Mayor, 2021 for counterevidence with Malay children); for instance, seven-year-olds were reportedly more likely to apply ME than six-year-olds (Merriman \& Kutlesic, 1993), who, in turn, showed stronger ME than four-year-olds (Davidson, Jergovic, Imami, \& Theodos, 1997). However, the developmental trajectories associated with the application of $\mathrm{AL}$ strategies have seldom been evaluated. Most studies have either recruited infants (Smith \& Yu, 2008; Vlach \& Johnson, 2013) or adults (Fitneva \& Christiansen, 2011; 
Kachergis, Yu, \& Shiffrin, 2012; Poepsel \& Weiss, 2014, 2016; Yu, Zhong, \& Fricker, 2012), with a few notable exceptions that compared adults and children of different age groups (Escudero, Mulak, \& Vlach, 2016; Fitneva \& Christiansen, 2017; Suanda et al., 2014). Older children appear to differ in the manifestation of word learning strategies; while four-year-olds learned word-object associations better if their initial guesses were correct, ten-year-olds performed independently from the accuracy of their initial guesses (Fitneva \& Christiansen, 2017). Yet, Suanda et al. (2014) did not find any differences in CSL performance between children aged five and seven years of age. In sum, the divergent characterisation of the effect of age on word learning strategies calls for further scrutiny - an objective of the present study.

The first aim of the present contribution is to test whether children establish and maintain multiple associations between words and objects - in line with AL accounts - or whether they only maintain a single association between words and objects - congruent with single-hypothesis accounts. To this end, the present study was carried out as an object-selection task, on an iPad. We presented children with two sets of "classical" ME trials ${ }^{1}$; in each set, two images were presented to the child - one illustrating a novel object and the other a name-known object - while a novel label is heard. It is noteworthy that ME trials present children with a potentially ambiguous learning situation, as the novel object is never ExPLICITLY (e.g., being displayed on its own) labelled using the novel word; as such, ME trials offer referential ambiguity. In fact, it is the precise intent of the current study to evaluate whether children would also map the novel label to the NAME-KNOWN object, thereby forming multiple word-object associations. To evaluate this possibility, we then introduce test trials, in which both name-known "distractors" are presented side-by-side while novel labels used during ME trials are being uttered.

Strict ME accounts would suggest that children should not display a preference for either object, as they should have rejected a second association to the name-known objects during ME trials. Other HT accounts would suggest that test trials falsify children's hypotheses that the novel object is the referent for the novel label (provided that this was their initial hypothesis) and would predict that children should randomly pick a new referent, as suggested by the propose-but-verify account (Medina et al., 2011; Trueswell et al., 2013; Woodard et al., 2016). In contrast, preference for the matching name-known object would indicate the children have NOT inhibited the formation of an additional association between the name-known object and the novel label, and that, instead, they have established multiple associations between words and objects, a view congruent with AL accounts (e.g., Smith \& Yu, 2008; Suanda et al., 2014; Vlach \& Johnson, 2013) ${ }^{2}$.

\footnotetext{
${ }^{1}$ Throughout the manuscript we will be using the shorthand "ME" to refer to trials in which children can use ME to fast-map, following a widely used terminology, e.g., Halberda (2003, 2006), Hansen and Markman (2009), Markman and Wachtel (1988), Mather and Plunkett (2009, 2010, 2011), and in line with Byers-Heinlein \& Werker's (2013) acknowledgement that disambiguation tasks (the term used in their paper) are also known as ME tasks in other studies.

${ }^{2}$ Please note that our design tests for the formation of multiple word-object associations (e.g., between a name-known object and a novel label). Additional test trials do not offer children the opportunity of further reducing the referential ambiguity-hence our study is not "cross-situational" per se (see also Smith et al., 2009 for a discussion of the cross-situational nature of statistical learning is some testing conditions). We see the formation of multiple, non-selective word-object associations a necessary pre-requisite to the full application of cross-situational statistical learning behaviour.
} 
The second aim of the present contribution is to evaluate whether children do, indeed, establish an association between the novel object and the novel label during ME trials, beyond "merely" displaying novelty preference for the novel object when hearing a novel label. Studies that investigated whether infants retain novel word associations following ME responses obtained varied results. Although Mervis and Bertrand (1994) found that 16- to 20-month-old infants displayed a novelty bias during ME trials and showed evidence of word learning during test trials, Horst and Samuelson (2008) found that 24-month-old infants were only able to display a novelty bias during the ME trials, but failed to retain the novel label to novel object mappings. Mather and Plunkett (2011) and Bion, Borovsky and Fernald (2013) conducted similar studies using eye-tracking paradigms. Mather and Plunkett's 16-month-old infants did not show an ME effect during ME trials, yet they learned novel word-object associations for one of the novel labels, while Bion et al.'s 24-month-olds displayed evidence of ME but failed to display evidence of retention. Together, this suggests that, while ME may lead to the formation of novel word-object associations, it is a fragile process that may be modulated by task difficulty and the age of participants.

The third aim is to describe the developmental trajectories associated with (a) novelty preference during ME trials, (b) the retention of novel word-object associations and (c) the potential formation of non-selective associations between novel labels and name-known objects. We focus on a wide age range, from 4 to 12 years of age, as this is when children acquire a major part of their vocabulary (Suanda et al., 2014, argues that "the rate of vocabulary growth during middle childhood is greater than during late infancy and toddlerhood, the period typically emphasised in word learning research" [p. 397]). In addition, this age range is of a considerable interest, as Fitneva and Christiansen (2017) reported that children go through qualitative changes in word learning behaviour, in CSL paradigms, between 4 and 10 years of age. Since the formation of additional word-object associations requires an increased memory load (Romberg \& Saffran, 2010), we expect that older children will establish more robust word-object associations than younger children.

In sum, the current study aims at evaluating whether children retain word-object associations formed during ME trials, as well as testing whether such word-object associations are unique - as suggested by single-hypothesis accounts - or non-selective (additional associations are formed between novel words and name-known objects) in line with $\mathrm{AL}$ accounts.

\section{Method}

\section{Participants}

One hundred and seventy-four children who participated in the Summer Scientist Week programme in The University of Nottingham were recruited in this study. Twenty-six participants who spoke more than one language at home were excluded from the analysis, to ensure that the results of the experiment would not be impacted by the number of languages spoken at home (e.g., Houston-Price et al., 2010). Of the remaining 148 English monolingual children, 65 were boys and 83 were girls. The children's age ranged from 4 years to 12 years $(M=7.36$ years, $S D=$ 2.06). There were 11 four-year-olds, 26 five-year-olds, 14 six-year-olds, 26 seven-year-olds, 25 eight-year-olds, 25 nine-year-olds, 9 ten-year-olds, 9 eleven-year-olds and 3 twelve-year-olds. Ethics approval was granted by The University of Nottingham Institutional Ethics board (JM190315). 


\begin{tabular}{|l|l|l|l|}
\hline Conditions & Image pairs & Auditory stimuli \\
\hline ME training & Find the modi! \\
\hline ME training & & & \\
\hline Word learning test & Find the dax! & \\
\hline Selectivity test & & & \\
\hline & & & \\
\hline
\end{tabular}

Figure 1. An example of different conditions.

\section{Stimuli}

The visual stimuli were images obtained from Frank, Sugarman, Horowitz, Lewis, and Yurovsky (2016). Each image had a resolution of 640 x 480 pixels and depicted an object on a grey background. Following Frank et al. (2016), the size of the images was set to fit comfortably on an iPad screen, with the width of the image set to 400 pixels and the height set to maintain the ratio of the image. Two novel labels, dax and modi, were used. Each novel label was associated with a novel object and a familiar object (see first two rows of Figure 1). The instruction to search for an object was "find the _.?", which was recorded by a female native English-speaker in an infant-directed manner.

\section{Procedure}

The experiment was carried out using an iPad. Before the start of the experiment, a warm-up task prompted children to tap on dots and smiley faces presented on the screen of the iPad. This task allowed children to familiarise themselves with the iPad. After the warm-up task, children were given instructions about the experiment; that they would be presented with two images, would be asked to find an object, and would have to respond by tapping one of the displayed images. Once the children had indicated that they had understood the procedure, the experiment began.

The experiment itself consisted of three conditions; ME training, a word learning test and a selectivity test (see Figure 1). In each trial, a pair of images was displayed on the screen while the instruction (the carrier sentence "find the __!") was played. The pair of images remained on screen until the child made a response by tapping on one of the 
images. There was a total of 8 trials in ME training ( 4 trials for each novel label), 4 trials in the word learning test ( 2 trials for each novel label) and in the selectivity test ( 2 trials for each novel label). Therefore, each child received a total of 16 experimental trials.

During ME training, the pair of images presented were a name-known and a novel object, while a novel label was uttered, in line with classical ME designs. Two image pairs, modi-cat and dax-carrot allowed every child to learn, via ME, two novel labels for two novel objects (see first two rows of Figure 1).

In the word learning test, children were tested on whether they had formed an association between a novel label and the corresponding novel object; an attempt to extend Mather and Plunkett's (2011) findings that ME can lead to the formation of word-object associations. Thus, the image pairs were made up of the two novel objects shown during ME training (see third row of Figure 1), whereas the auditory stimuli were the novel labels modi and dax.

In the selectivity test, the two NAME-KNOWN objects used during ME training (typically referred to as distractors in ME experiments) were displayed while, in each trial, one of the novel labels used during ME training was uttered (see last row of Figure 1). Target selection (that is, tapping on the name-known image from the same training pair) was taken as evidence that an association between the name-known object and a second label was not inhibited during ME training. The order of presentation of the word learning test and the selectivity test was counterbalanced across participants. A total of 73 children completed the word learning test before the selectivity test whereas the remaining 75 children completed the experiment in the reverse order.

Filler trials, consisting of pairs of familiar objects (different from those used in ME training), were presented between every two experimental trials, so as not to discourage the children by presenting only trials with novel labels. The familiar objects that were named were car, dog, lion and train. Attention getters were introduced between each condition so as to capture the attention of the participants.

\section{Analysis}

Response accuracy was analysed using a binomial mixed-effect logistic regression using the lme4 package in R (Version 3.3.1; Bates, Mächler, Bolker \& Walker, 2015), where selecting target objects was coded as 1 and distractor objects as 0 . The model included participants as random effects, condition (ME training, word learning test, selectivity test), age in months, test order (retention test or selectivity test first) and interaction between age and condition as fixed effects.

\section{Results}

Figure 2 illustrates the effect of age on response accuracy for all conditions, along with the $95 \%$ confidence intervals. The model revealed a main effect of condition (see Table 1). Post-hoc Tukey pairwise comparisons (corrected for multiple testing) between conditions revealed that children performed significantly better (all $p s<$ $.0001)$ in $\mathrm{ME}$ training $(M=.95, S D=.22)$ than in the word learning test $(M=.88$, $S D=.33)$ and in the selectivity test $(M=.60, S D=.49)$, and that they performed significantly better in the word learning test than in the selectivity test $(p<.0001)$. Children performed significantly above chance in all conditions ${ }^{3}$; in $\mathrm{ME}$ training

\footnotetext{
${ }^{3}$ Analysis of the intercept as produced by the minimal model (Response $\sim(1 \mid$ Participant $\left.)\right)$ for each condition.
} 


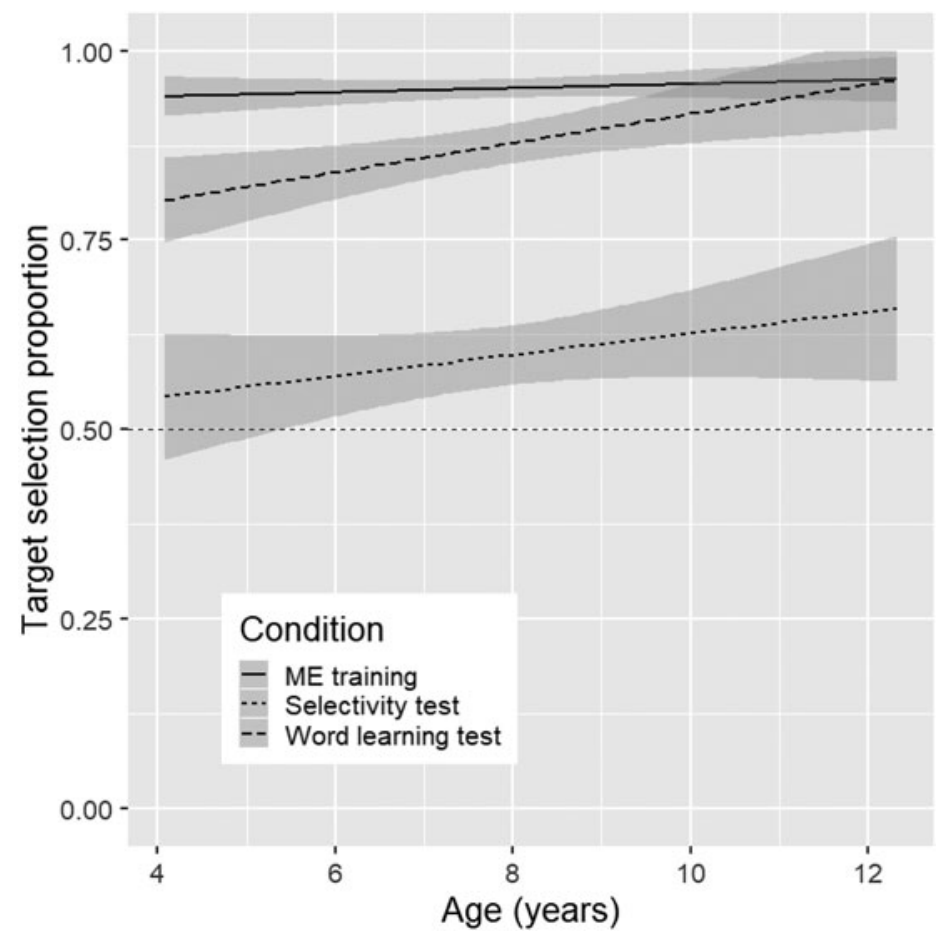

Figure 2. Linear regressions of the proportions of correct responses in each condition, as a function of age. Dotted horizontal line refers to chance at .50. Shaded areas indicate the $95 \%$ confidence intervals.

$(z=5.65, p<.0001)$, in the word learning test $(z=7.34, p<.0001)$, as well as in the selectivity test $(z=2.86, p<.004)$. The model also revealed a main effect of age, but no main effect of test order nor an effect of interaction between condition and age (see Table 1).

Exploratory analyses of the selectivity test using a binomial mixed-effect logistic regression, with participants as random effects and age in months, determined the age at which children display evidence of having formed associations between the novel labels and name-known objects. We conducted these analyses only for the selectivity test since there was a main effect of age but no age-condition interaction: yet, as is presented in Figure 2, children's performance in ME training and word learning test were clearly above chance. Hence, we look into the possibility that the main effect of age is driven by children's improvement in the selectivity test as they grow older. The intercept becomes significantly different from chance from 6.5 years of age and older $(z=2.02, p=.043)$. In other words, children from 6.5 years of age selected the matching name-known distractor significantly above chance.

\section{Discussion}

Children, and adults, frequently encounter new words when multiple potential referents are present in their visual scene. The nature of learning mechanisms in such ambiguous naming situations has been under scrutiny over decades and theories fall essentially into 
Table 1. Chi-square estimates, degrees of freedom and p-value of each fixed effect. The full model was Response $\sim(1 \mid$ Participant $)+$ Test_order + Age ${ }^{\star}$ Condition.

\begin{tabular}{lccc}
\hline Parameter & Chi-square & $\mathrm{df}$ & $\mathrm{p}$-value \\
\hline Condition & 375.59 & 2 & $<.0001$ \\
\hline Age & 4.19 & 1 & .041 \\
\hline Test order & 0.02 & 1 & .877 \\
\hline Interaction (Age*Condition) & 2.84 & 2 & .241 \\
\hline
\end{tabular}

two groups. The first group argues that learners establish and maintain a sINGLE HYPOTHESIS that is either confirmed or falsified with additional information (in which case a new, single, hypothesis is established). HT accounts can be considered to include early descriptions of learning mechanisms; the one-to-one mapping principle (Slobin, 1973), the uniqueness principle (Pinker, 1984; Wexler \& Culicover, 1980), as well as early accounts of ME (Markman \& Wachtel, 1988). These views have gained recent traction and are supported by additional evidence (Aravind et al., 2018; Berens et al., 2018; Medina et al., 2011; Trueswell et al., 2013; Woodard et al., 2016). In contrast, $\mathrm{AL}$ accounts suggest that children (Smith \& Yu, 2008; Suanda et al., 2014), and adults (Dautriche \& Chemla, 2014; Yu \& Smith, 2007) create and maintain a rich network of multiple associations between words and objects. The strength and relative importance of these associations are modulated when additional evidence is gained.

Yet, AL accounts typically present participants with sets of novel objects, thus making initial hypotheses, or associations, equiprobable. What if children are presented with an initial situation that favours one hypothesis? Would they still entertain the possibility that other hypotheses can hold, and create additional associations? To find out, we presented 4- to 12-year-old children with two sets of ME training trials, thus offering them the possibility of establishing two novel word-object associations. Subsequent test trials investigated whether children displayed evidence that they had also retained associations between the novel labels and the name-known distractors - a view congruent with AL accounts - or not - thus suggesting that they only tracked a single hypothesis.

Children displayed a strong bias for the novel object in ME training trials (in line with, e.g., Golinkoff, Hirsh-Pasek, Bailey, \& Wenger, 1992; Mather \& Plunkett, 2011). HT accounts would suggest that children have established an initial hypothesis; that the novel object is the referent for the novel label. Indeed, younger children (four and five years old) in the study failed to select the appropriate name-known distractor in the selectivity test, thus suggesting they have established and retained a single hypothesis, in line with propose-but-verify (Berens et al., 2018; Medina et al., 2011; Trueswell et al., 2013; Woodard et al., 2016) and ME accounts (Markman, 1990; Markman \& Wachtel, 1988).

Yet, children aged 6.5 years and older displayed a preference for the matching name-known object during selectivity test trials, when hearing novel labels used during ME training trials. This situation, with older children, does not sit well with HT accounts; when the initial hypothesis is falsified by test trials (the novel label is uttered while the novel object is not present), children should RANDOMLY generate a new hypothesis, as suggested by the propose-but-verify account (Berens et al., 2018; 
Medina et al., 2011; Trueswell et al., 2013; Woodard et al., 2016), and should not display a preference for either object presented during test trials.

In contrast, older children's preference for the matching name-known object when hearing novel labels suggest that they have established multiple word-object associations during ME training trials, despite their initial preference for the novel object. These non-selective associations between words and objects align well with a low-level association-based account of the CSL type that would also operate when NAME-KNOWN objects are presented to the child, and not only when novel objects are used. In other words, our results suggest that older children form multiple associations based on co-occurrences between words and multiple objects in their sight. Kachergis et al. (2012) and Smith, Smith, and Blythe (2011) argued that word learners only store more than one word-object associations if there are several potential referents for a label. Older children in our study appear to entertain that possibility. This pattern of results is also congruent with QUALITATIVE changes in CSL word learning reported by Fitneva and Christiansen (2017) between four-year olds and adults.

However, our results should also be examined from the perspective of children's regular, QUANTITATIVE, improvements in cognitive resources. For example, Vlach and DeBrock (2017) showed that children from two to five years of age differ in their ability to learn words in an ambiguous situation, as they differ in terms of their memory capacity. Romberg and Saffran (2010) highlighted the cognitive cost of creating novel word-object associations and Yurovsky and Frank (2015) shed light on the role of processing constraints on CSL performance. From this viewpoint, our design does not pose challenges to children aged six years and above; older children can form multiple word-object associations, in turn allowing them to remain flexible with word meanings and interpretations. In contrast, younger children possess more limited processing resources - in particular, when it comes to forming and retaining additional word-object associations-thus, merely retaining a single word-object association at a time.

An alternative explanation to our findings is that older children have formed associative relations between the co-occurring target and distractor (e.g., modi and cat) during ME training: hence, through cascaded activation, they selected the related familiar object in the selectivity test without having formed more than just one word-object associations. In other words, although older children were target-oriented in the selectivity test, this may not be inconsistent with HT accounts that word learners form single word-object mappings. Fixating related objects when the target is not present is reported in various eye-tracking studies that recruited either young children (Chow, Davies, \& Plunkett, 2017; Johnson \& Huettig, 2011) or adults (e.g., Huettig \& Altmann, 2005; Mirman \& Graziano, 2012). Future work will aim at teasing apart these two alternative possibilities, as the current design does not enable us to determine the exact mechanism that allows older children to be target-oriented in the selectivity test.

Yet, children of all ages performed well in the word learning test, thus providing evidence that they retained, at least temporarily, word-object associations formed during ME, in line with Mather and Plunkett (2011) and Mervis and Bertrand (1994). While children throughout the age range displayed evidence of retention of novel label to novel object associations, the positive age trend suggests that older children are stronger at learning novel names for novel objects. The parallel improvements with age (and the lack of interactions between age and condition) in forming associations between novel labels and both novel and name-known objects 
again points towards general cognitive improvements - older children form stronger word-object associations in general, and non-selective word-object associations in particular.

Children performed less robustly in the selectivity test than in the word learning test - which suggests that associations between novel labels and novel objects are stronger than associations between novel labels and name-known objects. Weaker performance in the selectivity test is again congruent with a low-level association-based account of the CSL type; the initial bias for the novel object in ME training trials establishes differing levels of attention towards each object. Higher attention towards an object (e.g., in our case, the novel object during ME training) leads to a stronger association between the word and the object, and weaker associations between the same word and other objects (in our case, the name-known object), in turn translating into a weaker performance in the selectivity test that reflects the initial relative preference for the novel object during ME training.

While we know that young children may have the capacity to form multiple word-object associations when potential referents are equated for novelty (e.g., from 12 months of age in Smith \& Yu, 2008), the youngest children in the present study (at four years of age) may not be able to overcome their initial preference for the novel object in the ME trials, nor "invest" sufficient time and cognitive resources to fixate and process the name-known distractor so that it can result in the formation of an additional word-object association. One possible reason is that our design presents a challenge to children - as the lack of contextual diversity (the same name-known object is always paired with the same novel object) is known to hinder cross-situational word learning (Kachergis, Shiffrin, \& Yu, 2009; Suanda \& Namy, 2012).

It should be noted that the absence of evidence of the formation of non-selective word-object associations, with the youngest children in our study, should not be interpreted as that they cannot form multiple associations (e.g., when all items are equally novel, as in most CSL designs). Similarly, the positive evidence that older children in our study form multiple word-object associations should not imply that older children, and adults, will not engage into leaner HT strategies at times - for example, when the complexity of the task requires it (Yurovsky \& Frank, 2015; Smith et al., 2011; Berens et al., 2018).

Furthermore, the observed preference for a novel object in a ME learning situation should not necessarily be taken as evidence for the rejection of additional associations between novel words and name-known objects. Indeed, the older children in our study appear to track multiple word-object associations, despite showing an initial preference for the novel object in ME training. This finding aligns with Kucker, McMurray and Samuelson's (2015) argument that two processes are at play; while FAST-MAPPING (e.g., using ME) helps the child disambiguate between objects in a given naming situation, a slower ACCUMULATION OF WORD-OBJECT MAPPINGS (e.g., through $\mathrm{AL}$ ) contributes to a gradual vocabulary learning via repeated exposure with words. Thus, although HT and AL accounts appear to be at odds with each other, older children in our study appear to rely on both strategies to their advantage. Although the formation of multiple mappings from a word to multiple objects in the visual scene cannot contribute to a reduction of the hypothesis space in ambiguous learning situations, it can provide the child with the capacity to entertain the possibility that multiple words can refer to the same object, a skill necessary to expand further her lexicon. 


\section{References}

Aravind, A., de Villiers, J., Pace, A., Valentine, H., Golinkoff, R., Hirsh-Pasek, K., Iglesias, A., \& Sweig Wilson, M. (2018). Fast mapping word meanings across trials: Young children forget all but their first guess. Cognition, 177, 177-188. https://doi.org/10.1016/j.cognition.2018.04.008

Bates, D., Mächler, M., Bolker, B., \& Walker, S. (2015). Fitting Linear Mixed-Effects Models using lme4. Journal of Statistical Software, 67(1), 1-48. Doi: 10.18637/jss.v067.i01

Berens, S. C., Horst, J. S., \& Bird, C. M. (2018). Cross-situational learning is supported by propose-but-verify hypothesis testing. Current Biology, 28, 1132-1136. Doi: 10.1016/j.cub.2018.02.042

Bion, R. A., Borovsky, A., \& Fernald, A. (2013). Fast mapping, slow learning: Disambiguation of novel word-object mappings in relation to vocabulary learning at 18, 24, and 30 months. Cognition, 126(1), 39-53. Doi: 10.1016/j.cognition.2012.08.008

Byers-Heinlein, K., \& Werker, J. F. (2009). Monolingual, bilingual, trilingual: Infants' language experience influences the development of a word-learning heuristic. Developmental Science, 12(5), 815-823. Doi: 10.1111/j.1467-7687.2009.00902.x

Chow, J., Davies, A. A., \& Plunkett, K. (2017). Spoken-word recognition in 2-year-olds: The tug of war between phonological and semantic activation. Journal of Memory and Language, 93, 104-134. Doi: 10.1016/j.jml.2016.08.004

Dautriche, I., \& Chemla, E. (2014). Cross-situational word learning in the right situations. Journal of Experimental Psychology: Learning, Memory, and Cognition, 40(3), 892-903. Doi: 10.1037/a0035657

Davidson, D., Jergovic, D., Imami, Z., \& Theodos, V. (1997). Monolingual and bilingual children's use of the mutual exclusivity constraint. Journal of Child Language, 24(1), 3-24. Doi: 10.1017/S0305000996002917

Escudero, P., Mulak, K. E., \& Vlach, H. A. (2016). Cross-situational learning of minimal word pairs. Cognitive Science, 40, 455-465. Doi: 10.1111/cogs.12243

Fitneva, S. A., \& Christiansen, M. H. (2011). Looking in the wrong direction correlates with more accurate word learning. Cognitive Science, 41, 141-161. Doi: 10.1111/cogs.12322

Fitneva, S. A., \& Christiansen, M. H. (2017). Developmental changes in cross-situational word learning: The inverse effect of initial accuracy. Cognitive Science, 41, 141-161. Doi: 10.1111/cogs.12322

Frank, M. C., Sugarman, E., Horowitz, A. C., Lewis, M. L., \& Yurovsky, D. (2016). Using tablets to collect data from young children. Journal of Cognition and Development, 17(1), 1-17. Doi: 10.1080/ 15248372.2015.1061528

Golinkoff, R. M., Hirsh-Pasek, K., Bailey, L. M., \& Wenger, N. R. (1992). Young children and adults use lexical principles to learn new nouns. Developmental Psychology, 28(1), 99-108. Doi: 10.1037/ 0012-1649.28.1.99

Horst, J. S., \& Samuelson, L. K. (2008). Fast mapping but poor retention by 24 -month-old infants. Infancy, 13(2), 128-157. Doi: 10.1080/15250000701795598

Houston-Price, C., Caloghiris, Z., \& Raviglione, E. (2010). Language experience shapes the development of the mutual exclusivity bias. Infancy, 15(2), 125-150. Doi: 10.1111/j.1532-7078.2009.00009.x

Huettig, F., \& Altmann, G. T. M. (2005). Word meaning and the control of eye fixation: Semantic competitor effects and the visual world paradigm. Cognition, 96, B23-B32. Doi: 10.1016/ j.cognition.2004.10.003

Johnson, E. K., \& Huettig, F. (2011). Eye movements during language-mediated visual search reveal a strong link between overt visual attention and lexical processing in 36-month-olds. Psychological Research, 75, 35-42. Doi: 10.1007/s00426-010-0285-4

Kachergis, G., Shiffrin, R., \& Yu, C. (2009). Frequency and contextual diversity effects in cross-situational word learning. In N. Taatgen, H. Van Rijn, J. Nerbonne, \& L. Schomaker (Eds.), Proceedings of the 31st Annual Meeting of the Cognitive Science Society (pp. 755-760), Austin, TX: Cognitive Science Society.

Kachergis, G., Yu, C., \& Shiffrin, R. M. (2012). An associative model of adaptive inference for learning word-referent mappings. Psychonomic Bulletin \& Review, 19(2), 317-324.

Kalashnikova, M., Escudero, P., \& Kidd, E. (2018). The development of fast-mapping and novel word retention strategies in monolingual and bilingual infants. Developmental Science, 21(6), e12674. Doi: $10.1111 /$ desc. 12674

Kalashnikova, M., Mattock, K., \& Monaghan, P. (2015). The effects of linguistic experience on the flexible use of mutual exclusivity in word learning. Bilingualism: Language and Cognition. Doi: 10.1017/ S1366728914000364 
Kucker, S. C., McMurray, B., \& Samuelson, L. K. (2015). Slowing down fast mapping: Redefining the dynamics of word learning. Child Development Perspectives, 9(2), 74-78. Doi: 10.1111/cdep.12110

Malone, S. A., Kalashnikova, M., \& Davis, E. M. (2016). Is it a name or a fact? Disambiguation of reference via exclusivity and pragmatic reasoning. Cognitive Science, 40, 2095-2107. Doi: 10.1111/ cogs. 12321

Markman, E. (1990). Constraints children place on word meanings. Cognitive Science, 14, 57-77. Doi: $10.1207 / \mathrm{s} 15516709 \operatorname{cog} 14014$

Markman, E., \& Wachtel, G. (1988). Children's use of mutual exclusivity to constrain the meanings of words. Cognitive Psychology, 20(2), 121-157. Doi: 10.1016/0010-0285(88)90017-5

Mather, E., \& Plunkett, K. (2011). Mutual exclusivity and phonological novelty constrain word learning at 16 months. Journal of Child Language, 38, 933-950. Doi: 10.1017/S0305000910000401

Medina, T. N., Snedeker, J., Trueswell, J. C., \& Gleitman, L. R. (2011). How words can and cannot be learned by observation. Proceedings of the National Academy of Sciences, 108(22), 9014-9019. Doi: 10.1073/pnas.1105040108

Merriman, W. E., \& Kutlesic, V. (1993). Bilingual and monolingual children's use of two lexical acquisition heuristics. Applied Psycholinguistics, 14(2), 229-249. Doi: 10.1017/S0142716400009565

Mervis, C. B., \& Bertrand, J. (1994). Acquisition of the novel name nameless category (N3C) principle. Child Development, 65, 1646-1662. Doi: 10.2307/1131285

Mirman, D., \& Graziano, K. M. (2012). Individual differences in the strength of taxonomic versus thematic relations. Journal of Experimental Psychology: General, 141(4), 601-609. Doi: 10.1037/a0026451

Pinker, S. (1984). Language learnability and language learning. Harvard University Press.

Poepsel, T. J., \& Weiss, D. J. (2014). Context influences conscious appraisal of cross situational statistical learning. Frontiers in Psychology, 5, 691. Doi: 10.3389/fpsyg.2014.00691

Poepsel, T. J., \& Weiss, D. J. (2016). The influence of bilingualism on statistical word learning. Cognition, 152, 9-19. Doi: 10.1016/j.cognition.2016.03.001

Quine, W. V. O. (1960). Word and object. MIT Press.

Romberg, A. R., \& Saffran, J. R. (2010). Statistical learning and language acquisition. Wiley Interdisciplinary Reviews: Cognitive Science, 1(6), 906-914. Doi: 10.1002/wcs.78

Sia, M. Y., \& Mayor, J. (2021). Syntactic cues help disambiguate objects referred to with count nouns: Illustration with Malay children. Child Development. Doi: 10.1111/cdev.13401

Slobin, D. I. (1973). Cognitive prerequisites for the development of grammar. In C. A. Ferguson, \& D. I. Slobin (Eds.), Studies of child language development (pp. 175-208). Holt, Rinehart, \& Winston.

Smith, K., Smith, A. D., \& Blythe, R. A. (2011). Cross-situational learning: An experimental study of word-learning mechanisms. Cognitive Science, 35(3), 480-498. Doi: 10.1111/j.1551-6709.2010.01158.x

Smith, L., \& Yu, C. (2008). Infants rapidly learn word-referent mappings via cross-situational statistics. Cognition, 106(3), 1558-1568. Doi: 10.1016/j.cognition.2007.06.010

Suanda, S. H., Mugwanya, N., \& Namy, L. L. (2014). Cross-situational statistical word learning in young children. Journal of Experimental Child Psychology, 126, 395-411. Doi: 10.1016/j.jecp.2014.06.003

Suanda, S. H., \& Namy, L. L. (2012). Detailed behavioral analysis as a window into cross-situational word learning. Cognitive Science, 36, 545-559. Doi: 10.1111/j.1551-6709.2011.01218.x

Trueswell, J. C., Medina, T. N., Hafri, A., \& Gleitman, L. R. (2013). Propose but verify: Fast mapping meets cross-situational word learning. Cognitive Psychology, 66(1), 126-156. Doi: 10.1016/ j.cogpsych.2012.10.001

Vlach, H. A., \& DeBrock, C. A. (2017). Remember dax? Relations between children's cross-situational word learning, memory, and language abilities. Journal of Memory and Language, 93, 217-230. Doi: 10.1016/j.jml.2016.10.001

Vlach, H. A., \& Johnson, S. P. (2013). Memory constraints on infants' cross-situational statistical learning. Cognition, 127, 375-382. Doi: 10.1016/j.cognition.2013.02.015

Wexler, K., \& Culicover, P. (1980). Formal Principles of Language Acquisition. MIT Press.

Woodard, K., Gleitman, L. R., \& Trueswell, J. C. (2016). Two- and three-year-olds track a single meaning during word learning: Evidence for propose-but-verify. Language Learning and Development, 12(3), 252-261. Doi: 10.1080/15475441.2016.1140581

Yu, C., \& Smith, L. B. (2007). Rapid word learning under uncertainty via cross-situational statistics. Psychological Science, 18(5), 414-420. Doi: 10.1111/j.1467-9280.2007.01915.x 
Yu, C., Zhong, Y., \& Fricker, D. (2012). Selective attention in cross-situational statistical learning: Evidence from eye tracking. Frontiers in Psychology, 3(148), 1-16. Doi: 10.3389/fpsyg.2012.00148

Yurovsky, D., \& Frank, M. C. (2015). An integrative account of constraints on cross-situational learning. Cognition, 145, 53-62. Doi: 10.1016/j.cognition.2015.07.013

Cite this article: Sia MY, Mayor J (2022). Improvements of Statistical Learning Skills Allow Older Children to Go Beyond Single-Hypothesis Testing When Learning Words. Journal of Child Language 49, 1268-1280. https://doi.org/10.1017/S0305000921000532 